REVISTA DE LA

UNIÓN MATEMÁTICA ARGENTINA

Vol. 59, No. 2, 2018, Pages 415-429

Published online: June 8, 2018

\title{
QUESTIONS AND CONJECTURES ON EXTREMAL HILBERT SERIES
}

\author{
RALF FRÖBERG AND SAMUEL LUNDQVIST
}

\begin{abstract}
Given an ideal of forms in an algebra (polynomial ring, tensor algebra, exterior algebra, Lie algebra, bigraded polynomial ring), we consider the Hilbert series of the factor ring. We concentrate on the minimal Hilbert series, which is achieved when the forms are generic. In the polynomial ring we also consider the opposite case of maximal series. This is mainly a survey article, but we give a lot of problems and conjectures. The only novel results concern the maximal series in the polynomial ring.
\end{abstract}

\section{INTRODUCTION}

The Hilbert series of a graded commutative algebra is an important invariant in commutative algebra and algebraic geometry. It has since long been known what the Hilbert series of the quotient ring $k\left[x_{1}, \ldots, x_{n}\right] / I$ can be for a homogeneous ideal $I$ in a polynomial ring over a field $k[23]$. A much harder question is: If $I$ is generated by forms $f_{1}, \ldots, f_{r}$ of degrees $d_{1}, \ldots, d_{r}$, what can the Hilbert series be? Not much is known about this. This question is relevant also for other kinds of graded algebras, and in these cases, even less is known.

It is not hard to see that, given a degree sequence $d_{1}, \ldots, d_{r}$, one can construct an algebra with a minimal series in the lexicographical sense, by choosing the forms $f_{i}$ to be generic. (We say that $\sum a_{i} z^{i}$ is smaller than $\sum b_{i} z^{i}$ lexicographically if for the smallest $i$ for which $a_{i} \neq b_{i}$, we have $a_{i}<b_{i}$.) A form $f$ in a graded $k$-algebra $R$ is generic if the coefficients are algebraically independent over the prime field of $k$. By generic forms we mean that the coefficients of all forms are algebraically independent. That choosing the $f_{i}$ 's as generic forms really gives a minimal series follows from the fact that a non-trivial relation of generic forms specializes to a relation of specific forms. In the commutative case, this is Lemma 1 in [12.

We call an algebra which is the quotient of an ideal generated by generic forms generic. We denote the Hilbert series $\sum_{i \geq 0} \operatorname{dim}_{k} R_{i} z^{i}$ of a graded $k$-algebra $R$ by $R(z)$, and we will assume that $k=\mathbb{C}$ unless otherwise stated.

In Section 2 we consider minimal and maximal Hilbert series for factor rings of polynomial rings and some related properties. In the subsequent sections we consider problems related to minimality for tensor algebras, Lie algebras, exterior

2010 Mathematics Subject Classification. 13D40, 13P10, 16S30, 16Z05, 17B35. 
algebras, and bigraded algebras. We finish with some comments on finite characteristic.

\section{Polynomial Rings}

Let $R=k\left[x_{1}, \ldots, x_{n}\right] /\left(f_{1}, \ldots, f_{r}\right), f_{i}$ forms of degree $d_{i}, i=1, \ldots, r$. It is shown by Fröberg and Löfwall [14, that there is only a finite number of Hilbert series, and that there is an open nonempty subspace of the space of coordinates for the $f_{i}$ 's on which the Hilbert series is constant and minimal. The idea is that given $\left(n, d_{1}, \ldots, d_{r}\right)$, there is a bound for the Castelnuovo-Mumford regularity, and for each degree the algebras with nonminimal dimension constitute a closed set. Since one only has to check a finite set of dimensions, the algebras with nonminimal dimension in those degrees is closed. Thus there exists a truly (i.e., coefficientwise) minimal series, not only minimal in the lexicographical sense.

There is a longstanding conjecture due to the first author for this minimal Hilbert series, see [12].

Conjecture 1. $R(z)=\left(\prod_{i=1}^{r}\left(1-z^{d_{i}}\right) /(1-z)^{n}\right)_{+}$, where $\left(\sum_{i \geq 0} a_{i} z^{i}\right)_{+}=\sum_{i \geq 0} b_{i} z^{i}$, where $b_{i}=a_{i}$ if $a_{j} \geq 0$ for all $j \leq i$ and $b_{i}=0$ otherwise.

The conjecture is proved if $r \leq n$ (complete intersection case), if $n \leq 2$ [12, if $n=3$ by Anick [4, and if $r=n+1$, it follows from [35]. (In fact Stanley proves something more, see below.) It is known that the true series is larger or equal to the conjectured, which gives that if one finds an algebra with the conjectured series for some $\left(n, r, d_{1}, \ldots, d_{r}\right)$, then the conjecture is proved for these values. There are also some further special results when all $d_{i}$ are equal, $d_{i}=d$ for all $i$. If $(n, d, r)=(4, d, 4)$, by Backelin and Oneto [6]; $(n, d, r)=(4, d, r)$ for $d=4,6,8,9$ and $(n, d, r)=(5,4, r)$, by Nicklasson [33]; $(n, 2,3), n \leq 11$ and $(n, 3, r), n \leq 8$, by Fröberg and Hollman [13. It is also proved by Hochster and Laksov that the formula is correct in the first nontrivial degree $\min \left\{d_{i}\right\}+1$, [19]. The last result has been generalized in two different ways to an interval $t \leq d+d^{\prime}, d^{\prime}<d$ (where there are no Koszul relations) if all generators are of degree $d$. The first, by Aubry in [5], is only depending on $n$. The conjectured series is correct in degrees $t \leq d+d^{\prime}$ if

$d \geq 2 d^{\prime}(n-1) / \sqrt[n-1]{(n-1) !}-d^{\prime}+\left(d^{\prime}\right)^{2} / \sqrt[n-2]{(n-2) !}+(n-1)^{2} / \sqrt[n-1]{(n-1) !}-n+5$.

There is also a generalization only depending on $r$ by Migliore and Miró-Roig. The conjectured series is correct in degrees $t \leq d+d^{\prime}$ if $\left(\begin{array}{c}d+d^{\prime}+2 \\ d+d^{\prime}\end{array}\right) \geq r\left(\begin{array}{c}d^{\prime}+2 \\ d^{\prime}\end{array}\right)$ in [24]. We also mention that the conjecture is proved in many cases by Nenashev [29].

Here are some open questions. A positive answer to Question 2.4 would give a positive answer to Question 2.3. which would give a positive answer to Question 2.2 The rings in Question 2.4 are studied in [8].

Question 2.1. Let $f_{1}, \ldots, f_{r}$ be generic forms, $r \geq n+1$. Stanley showed that the Hilbert series of $k\left[x_{1}, \ldots, x_{n}\right] /\left(f_{1}, \ldots, f_{n+1}\right)$ and $k\left[x_{1}, \ldots, x_{n}\right] /\left(x_{1}^{d_{1}}, \ldots, x_{n}^{d_{n}}, f_{n+1}\right)$ are equal, where $\operatorname{deg}\left(f_{i}\right)=d_{i}$. Does the same hold for $k\left[x_{1}, \ldots, x_{n}\right] /\left(f_{1}, \ldots, f_{r}\right)$ and $k\left[x_{1}, \ldots, x_{n}\right] /\left(x_{1}^{d_{1}}, \ldots, x_{n}^{d_{n}}, f_{n+1}, \ldots, f_{r}\right)$ when $r \geq n+1$ ? If all $d_{i}=d$ ? 
Question 2.2. Let $g_{1}, \ldots, g_{r}$ be generic forms of degree $d>1$, in $k\left[x_{1}, \ldots, x_{n}\right]$. Is the Hilbert series of $k\left[x_{1}, \ldots, x_{n}\right] /\left(g_{1}^{k}, \ldots, g_{r}^{k}\right)$ the same as the Hilbert series of $k\left[x_{1}, \ldots, x_{n}\right] /\left(f_{1}, \ldots, f_{r}\right), f_{i}$ generic of degree $d k$ ? This is conjectured in 33], and proved in some cases. The corresponding question for $d=1$ is not true, see Section 2.2 and particularly Question 2.14.

Question 2.3. Let $\mathbf{d}=\left(d_{1}, \ldots, d_{k}\right)$ be natural numbers with $d_{1}+\cdots+d_{k}=d$. Let $g_{i}$ be generic of degree $d_{i}$ and let $h_{i}=\prod_{i=1}^{k} g_{i}$. Does $k\left[x_{1}, \ldots, x_{n}\right] /\left(h_{1}, \ldots, h_{r}\right)$ have the same Hilbert series as $k\left[x_{1}, \ldots, x_{n}\right] /\left(f_{1}, \ldots, f_{r}\right), f_{i}$ generic of degree $d$ if $\mathbf{d} \neq(1,1, \ldots, 1)$ ?

Question 2.4. Let $\mathbf{d}=\left(d_{1}, \ldots, d_{k}\right)$ be natural numbers with $d_{1}+\cdots+d_{k}=d$, and let $g_{i}=l_{i 1}^{d_{1}} \cdots l_{i k}^{d_{k}}$, where $l_{i j}$ are generic linear forms. Does $k\left[x_{1}, \ldots, x_{n}\right] /\left(g_{1}, \ldots, g_{k}\right)$ have the same Hilbert series as $k\left[x_{1}, \ldots, x_{n}\right] /\left(f_{1}, \ldots, f_{r}\right), f_{i}$ generic of degree $d$ if $\mathbf{d} \neq(1,1, \ldots, 1)$ ?

Question 2.5. When $r \geq n$, the expression $\left(\prod_{i=1}^{r}\left(1-z^{d_{i}}\right) /(1-z)^{n}\right)+$ is a polynomial in $z$. What are the coefficients? What is the degree?

Question 2.6. Let $k\left[y_{1}, \ldots, y_{n}\right]$ be the ring of differential operators acting on $k\left[x_{1}, \ldots, x_{n}\right]$. Let $f$ be a generic form in $k\left[x_{1}, \ldots, x_{n}\right]$ of degree $d$ and let $m_{1}, \ldots, m_{r}$ be quadratic monomials in $k\left[y_{1}, \ldots, y_{n}\right]$. Does $k\left[x_{1}, \ldots, x_{n}\right] /\left(m_{1} . f, \ldots, m_{r} . f\right)$ have the same series as $k\left[x_{1}, \ldots, x_{n}\right] /\left(f_{1}, \ldots, f_{r}\right)$, the $f_{i}$ 's generic forms of degree $d-2$ ?

Question 2.7. Let $f_{1}, \ldots, f_{r}$ be generic forms of degree $d$ with $r>n$. When is the Hilbert series of $k\left[x_{1}, \ldots, x_{n}\right] / I^{s}$ equal to the Hilbert series of $k\left[x_{1}, \ldots, x_{n}\right] / J$, where $J$ is generated by $\left(\begin{array}{c}s+r-1 \\ r-1\end{array}\right)$ generic forms of degree ds? Related questions were studied in [7].

Maximal Hilbert series. We now turn to the opposite problem. What is the coefficientwise maximal (if it exists) Hilbert series that an algebra with given degree sequence can attain?

It is well known that the upper bound for the Hilbert series in the uniform degree case is achieved when $I$ is a lex segment ideal and follows directly from the characterization of Hilbert series due to Macaulay [23. However, we are not aware of any results in the mixed degree case. We can give an answer in the case of two variables.

Theorem 1. Let $k$ be any field and let $f_{1}, \ldots, f_{r}$ be forms in $k[x, y]$ of degrees $d_{1} \geq \cdots \geq d_{r}$ such that $\left(f_{1}, \ldots, f_{r}\right)$ is a minimally generated ideal. Then

$$
k[x, y] /\left(f_{1}, \ldots, f_{r}\right)(z) \leq \frac{1+z+\cdots+z^{d_{r}-1}-\left(z^{d_{r-1}}+z^{d_{r-2}}+\cdots+z^{d_{1}}\right)}{1-z} .
$$

Proof. Without loss of generality, we can assume that $\left(\operatorname{lm}\left(f_{1}\right), \ldots, \operatorname{lm}\left(f_{r}\right)\right)=\left(m_{1}\right.$, $\left.\ldots, m_{r}\right)$ is a minimally generated ideal of leading monomials with respect to some monomial ordering. It is well known that

$$
k[x, y] /\left(f_{1}, \ldots, f_{r}\right)(z) \leq k[x, y] /\left(\operatorname{lm}\left(f_{1}\right), \ldots, \operatorname{lm}\left(f_{r}\right)\right)(z),
$$


so it is enough to show that

$$
R(z) \leq \frac{1+z+\cdots+z^{d_{r}-1}-\left(z^{d_{r-1}}+z^{d_{r-2}}+\cdots+z^{d_{1}}\right)}{1-z},
$$

where $R=k[x, y] /\left(m_{1}, \ldots, m_{r}\right)$.

For each $i, i=1, \ldots, r$, we divide the monomials $m_{1}, \ldots, m_{r}$ into three disjoint groups. Let the first group consist of $m_{i}$ only. Let the second group consist of the monomials having $x$-degree less than $m_{i}$, and let the third group consist of the monomials having $y$-degree less than $m_{i}$. Since $\left(m_{1}, \ldots, m_{r}\right)$ is minimally generated, it follows that the three groups form a partition of $\left\{m_{1}, \ldots, m_{r}\right\}$ for each $i$.

Fix one $i$. The Hilbert series of $\left(m_{i}\right)$ is equal to $\frac{z^{d_{i}}}{(1-z)^{2}}$. Denote by $B$ and $C$ the second and the third group respectively, and write $B=\left\{m_{j_{1}}, \ldots, m_{j_{s}}\right\}$, $C=\left\{m_{k_{1}}, \ldots, m_{k_{t}}\right\}$. In the ideal $\left(m_{j_{h}}\right)$, there is an infinite set $I_{h}=\left\{m_{j_{h}}, m_{j_{h}} \cdot x\right.$, $\left.m_{j_{h}} \cdot x^{2}, \ldots\right\}$ of monomials. Likewise, in the ideal $\left(m_{k_{h}}\right)$, there is an infinite set $J_{h}=\left\{m_{k_{h}}, m_{k_{h}} \cdot y, m_{k_{h}} \cdot y^{2}, \ldots\right\}$ of monomials. Each element in $B$ has a unique $x$-degree, and each element in $C$ has a unique $y$-degree, so the $s+t+1=r$ sets $I_{1}, I_{2}, \ldots, I_{s}, J_{1}, J_{2}, \ldots, J_{t},\left\{\right.$ monomials in $\left.\left(m_{i}\right)\right\}$ are disjoint.

Thus, the Hilbert series of $\left(m_{1}, \ldots, m_{r}\right)$ is coefficientwise greater than or equal to $\max \left(h_{i}\right)$, where

$$
\begin{aligned}
h_{i} & =\frac{z^{d_{i}}}{(1-z)^{2}}+\frac{z^{d_{1}}+\cdots+z^{d_{i-1}}+z^{d_{i+1}}+\cdots+z^{d_{r}}}{1-z} \\
& =\frac{z^{d_{1}}+\cdots+z^{d_{r}}-\left(+z^{d_{1}+1}+\cdots+z^{d_{i-1}+1}+z^{d_{i+1}+1}+\cdots+z^{d_{r}+1}\right)}{(1-z)^{2}} .
\end{aligned}
$$

For $i \geq 2$, we have

$$
h_{i}-h_{i-1}=\frac{-z^{d_{i-1}+1}+z^{d_{i}+1}}{(1-z)^{2}} .
$$

Since $d_{i-1} \geq d_{i}$, the coefficients in the series $h_{i}-h_{i-1}$ are non-negative. This shows that $h_{1} \leq \cdots \leq h_{r}$ coefficientwise, and especially, that the Hilbert series of $\left(m_{1}, \ldots, m_{r}\right)$ is greater than or equal to $h_{r}$, so

$$
\begin{aligned}
R(z) & \leq \frac{1}{(1-z)^{2}}-\left(\frac{z^{d_{r}}}{(1-z)^{2}}+\frac{z^{d_{r-1}}+z^{d_{r-2}}+\cdots+z^{d_{1}}}{1-z}\right) \\
& =\frac{1+z+\cdots+z^{d_{r}-1}}{1-z}-\frac{z^{d_{r-1}}+z^{d_{r-2}}+\cdots+z^{d_{1}}}{1-z} .
\end{aligned}
$$

We now give an explicit construction of an ideal which attains the maximal possible Hilbert series. We first remark that in Theorem 1, we actually have $d_{r} \geq r-1$. Indeed, suppose that $I=\left(\operatorname{lm}\left(f_{1}\right), \ldots, \operatorname{lm}\left(f_{r}\right)\right) \subseteq k[x, y]$ is minimally generated. Let $m=x^{a} y^{b}$ be a monomial of least degree in $I$. By the minimality assumption, each other generator either has $x$-degree less than $a$, or $y$-degree less than $b$. Moreover, two different generators can not have the same $x$ - or $y$-degree. So there are at most $a+b+1$ monomials in $I$. It follows that if $I$ is minimally generated by forms of degrees $d_{1} \geq \cdots \geq d_{r}$, then $d_{r} \geq r-1$ (and if $d_{r}=r-1$, then $k[x, y] / I$ is artinian). 
Theorem 2. Let $d_{1} \geq \cdots \geq d_{r} \geq r$ and let $I=\left(x^{d_{1}}, x^{d_{2}-1} y^{1}, x^{d_{3}-2} y^{2}, \ldots\right.$, $\left.x^{d_{r}-(r-1)} y^{r-1}\right)$. Then the Hilbert series of $k[x, y] / I$ is equal to

$$
\frac{1+z+\cdots+z^{d_{r}-1}-\left(z^{d_{r-1}}+z^{d_{r-2}}+\cdots+z^{d_{1}}\right)}{1-z} .
$$

Proof. The Hilbert series of the ideal $\left(x^{d_{r}-(r-1)} y^{r-1}\right)$ is equal to $\frac{z^{d_{r}}}{(1-z)^{2}}$. Next, consider the ideal $\left(x^{d_{r-1}-(r-2)} y^{r-2}, x^{d_{r}-(r-1)} y^{r-1}\right)$. This ideal consists of the monomials in $\left(x^{d_{r}-(r-1)} y^{r-1}\right)$ together with the monomials of the form $x^{i} y^{r-2}$ with $i \geq d_{r-1}-(r-2)$, so the Hilbert series is equal to $\frac{z^{d_{r}}}{(1-z)^{2}}+\frac{z^{d_{r-1}}}{1-z}$. Repeating this argument, we arrive at the conclusion that the Hilbert series of $I$ is equal to

$$
\frac{z^{d_{r}}}{(1-z)^{2}}+\frac{z^{d_{r-1}}+z^{d_{r-2}}+\cdots+z^{d_{1}}}{1-z} .
$$

It follows that the Hilbert series of $k[x, y] / I$ equals

$$
\begin{aligned}
\frac{1}{(1-z)^{2}}-\left(\frac{z^{d_{r}}}{(1-z)^{2}}+\right. & \left.\frac{z^{d_{r-1}}+z^{d_{r-2}}+\cdots+z^{d_{1}}}{1-z}\right) \\
& =\frac{1+z+\cdots+z^{d_{r}-1}}{1-z}-\frac{z^{d_{r-1}}+z^{d_{r-2}}+\cdots+z^{d_{1}}}{1-z} .
\end{aligned}
$$

Consider now the three variable case. It is not clear how to choose the monomials in order to maximize the series. In fact, it is not even clear that there is a maximal series in the coefficientwise sense. Let $\mathbf{d}=(5,4,3,2)$. Natural choices for maximizing the series would be $I=\left(x^{5}, x^{3} y, x^{2} z, y^{2}\right)$ or $J=\left(x^{2}, x y^{2}, x z^{3}, y^{5}\right)$, where the ideal $I$ is constructed as follows. We start with degree five. We choose $x^{5}$ since it is the biggest monomial with respect to Lex, and we then proceed in descending degree order, picking $x^{3} y$ since it is the biggest monomial with respect to Lex which is not in $\left(x^{5}\right)$, and so on. The monomials in $J$ are chosen in the same manner, but with respect to ascending degree order.

It turns out that $k[x, y, z] / I(z)<k[x, y, z] / J(z)$ coefficientwise, but in fact, there is another choice that gives a minimal series, namely $K=\left(x^{5}, x^{3} y, x y^{2}, x z\right)$. Here we have chosen the generators with respect to reverse Lex and in descending degree order. In [11, revlex ideals and minimal Betti numbers were studied, and we believe that there could be a connection to the problem that we consider.

Question 2.8. Is there a maximal Hilbert series when $n \geq 3$ in the coefficientwise sense? If so, what is it?

2.1. Resolutions. There has been some work on the resolution of ideals generated by generic forms by Migliore and Miró-Roig [24, 25], and by Pardue and Richert [31, 32]. Let $R=k\left[x_{1}, \ldots, x_{n}\right] / I=S / I$, and let $\beta_{i, j}=\operatorname{dim}_{k}\left(\operatorname{Tor}_{i}^{S}(R, k)\right)_{j}$. Then we have that $R(z)=\sum_{i=1}^{n}(-1)^{i} \beta_{i, j} z^{j} /(1-z)^{n}$. If $I$ is minimally generated by generic elements $f_{1}, \ldots, f_{r}, \operatorname{deg}\left(f_{i}\right)=d_{i}$, then $\beta_{i, j}=0$ if $j-i \geq e$, where $e$ is the Castelnuovo regularity of $R$, so $R_{e} \neq 0$ but $R_{e+1}=0$ in the artinian case. It is shown that the resolution agrees with the Koszul resolution of $\left(f_{1}, \ldots, f_{r}\right)$ in degrees $j-i \leq e-2$ (and also in degree $j-i=e-1$ if the first nonpositive coefficient 
in $\prod_{i=1}^{r}\left(1-z^{d_{i}}\right) /(1-z)^{n}$ is 0$)$. They also show that if $f_{1}, \ldots, f_{n+1}$ are generic forms, $\operatorname{deg}\left(f_{i}\right)=d_{i}$, which minimally generate $\left(f_{1}, \ldots, f_{n+1}\right)$ in $k\left[x_{1}, \ldots, x_{n}\right]$, the first nonpositive coefficient is 0 if and only if $\sum_{i=1}^{n+1} d_{i}$ is odd. It is natural to conjecture that the only instances when $\beta_{i, j}=\beta_{i_{2}, j}$ should come from Koszul relations (and in fact this was conjectured in [21]). This is shown to be false. A small counterexample from [24] is the resolution of four forms of degrees $5,5,5$, and 7 in $S=k[x, y, z]$. The minimal resolution has the form

$$
0 \rightarrow S[-12]^{4} \oplus S[-11] \rightarrow S[-10]^{7} \oplus S[-11] \rightarrow S[-5]^{3} \oplus S[-7] \rightarrow S \rightarrow S / I \rightarrow 0 .
$$

Here the "ghost terms" $S[-11]$ are not explained through Koszul relations and cannot be predicted from the Hilbert series. In case all relations have the same degree, there should be no ghost terms.

If all generators have the same degree, there exists a ghost term in the minimal resolution if we have $\beta_{i_{1}, j} \neq 0$ and $\beta_{i_{2}, j} \neq 0$ with $i_{1} \neq i_{2}$ for some $j$. If there are no ghost terms, then the Betti numbers can be determined from the Hilbert series, and the resolution is called pure.

Conjecture 2 ([25, Conjecture 5.8]). Let $I \subset R=k\left[x_{1}, \ldots, x_{n}\right]$ be the ideal of $d>n$ generically chosen forms of the same degree. Then there is no redundant term in the minimal free resolution of $R / I$. Consequently, the minimal free resolution is the minimum one consistent with the Hilbert function.

Theorem 3 ([25]). Suppose that $R$ is an almost complete intersection, $r=n+1$, generated by generic forms. Then Conjecture 2 is true when $n=3$, when both $n$ and $d$ are even, and when $(n, d)=(4,3)$.

We can add

Theorem 4. Conjecture 2 is true for any $r$ when $d=2, n \leq 5$; when $d=3, n \leq 4$; and when $d=4, n=3$.

This is proved with computer calculations in Macaulay2, see [18.

There is an example in [31] showing that the space of generic ideals with minimal Betti numbers is strictly smaller than the space of ideals with generic Hilbert function. Let $I$ be an ideal generated by five generic forms of degree four in $k[x, y, z, u]$, and let $J=\left(x^{4}, y^{4}, z^{4}, u^{4},(x+y+z+u)^{4}\right)$. Then both $k[x, y, z, u] / I$ and $k[x, y, z, u] / J$ have generic series. The resolution of $R=k[x, y, z, u] / J=S / J$ is

$$
0 \rightarrow S[-9]^{6} \rightarrow S[-8]^{9} \oplus S[-7]^{9} \rightarrow S[-6]^{16} \rightarrow S[-4]^{5} \rightarrow S \rightarrow S \rightarrow S / I \rightarrow 0 .
$$

The resolution of $S / J$ has ghost terms $S[-7]$ in homological degrees 2 and 3 , and $S[-8]$ in homological degrees 3 and 4 , but there are no ghost terms in the resolution of $S / I$. Here is another example we have come across. Let $R=$ $k\left[x_{1}, x_{2}, x_{3}, x_{4}\right] /\left(f_{1}, \ldots, f_{8}\right), f_{i}$ generic of degree 3 , and $T=\frac{k\left[x_{1}, x_{2}, x_{3}, x_{4}\right]}{\left(x_{1}^{3}, x_{2}^{3}, x_{3}^{3}, x_{4}^{3},\left(x_{1}+x_{2}+x_{3}\right)^{3},\left(x_{1}+x_{2}+x_{4}\right)^{3},\left(x_{1}+x_{3}+x_{4}\right)^{3},\left(x_{2}+x_{3}+x_{4}\right)^{3}\right)}$.

Then $R$ and $T$ have Hilbert series as in Conjecture $1, R$ has minimal Betti numbers but $T$ has ghost terms $S[-5]^{3}$ in homological degrees 2 and 3 , and $S[-6]^{6}$ in 
homological degrees 3 and 4 . We believe that this is the first example of a series; see the questions below.

Question 2.9. Let $f_{1}, \ldots, f_{r}$ be generic forms of degree $d$. Do $k\left[x_{1}, \ldots, x_{n}\right] /\left(f_{1}\right.$, $\left.\ldots, f_{r}\right)$ and $k\left[x_{1}, \ldots, x_{n}\right] /\left(x_{1}^{d}, \ldots, x_{n}^{d}, f_{n+1}, \ldots, f_{r}\right)$ have the same Betti numbers?

Question 2.10. Does $k[x, y, z, u] /\left(x^{d}, y^{d}, z^{d}, u^{d},(x+y+z+u)^{d}\right)$ have minimal Betti numbers if $d \neq 4$ ?

Question 2.11. Let $R=k\left[x_{1}, x_{2}, x_{3}, x_{4}\right] /\left(f_{1}, \ldots, f_{8}\right), f_{i}$ generic of degree $d \geq 3$, and let $T=k\left[x_{1}, x_{2}, x_{3}, x_{4}\right] /\left(x_{1}^{d}, x_{2}^{d}, x_{3}^{d}, x_{4}^{d},\left(x_{1}+x_{2}+x_{3}\right)^{d},\left(x_{1}+x_{2}+x_{4}\right)^{d},\left(x_{1}+x_{3}+\right.\right.$ $\left.\left.x_{4}\right)^{d},\left(x_{2}+x_{3}+x_{4}\right)^{d}\right)$. Is it true that $R$ and $T$ have Hilbert series as in Conjecture 1 . $R$ has minimal Betti numbers, and $T$ has ghost terms $S[-(2 d-1)]^{3}$ in homological degrees 2 and 3, and $S[-2 d]^{d+3}$ in homological degrees 3 and 4? (This is true for $d \leq 8$.)

Question 2.12. Let $R=k\left[x_{1}, x_{2}, x_{3}, x_{4}\right] /\left(f_{1}, \ldots, f_{8}\right), f_{i}$ generic of degree $d \geq 2$, and let $T=k\left[x_{1}, x_{2}, x_{3}, x_{4}\right] / I$, where $I$ is generated by the 8 forms $\left(x_{1} \pm x_{2} \pm x_{3} \pm\right.$ $\left.x_{4}\right)^{d}$. Is $T(z)-R(z)=\left(\begin{array}{l}d \\ 2\end{array}\right) z^{2(d-1)}$ ?

2.2. Connections to the Lefschetz properties. A graded algebra $A$ has the maximal rank property (MRP) if for any $d$, the map $A_{i} \stackrel{f}{\rightarrow} A_{i+d}$ has maximal rank, i.e., is injective or surjective for all $i$, if $f$ is a generic form of degree $d$.

The following lemma is easily proved.

Lemma 5 ([12, Lemma 4]).

$$
\left(\left(1-z^{d_{r+1}}\right)\left(\prod_{i=1}^{r}\left(1-z^{d_{i}}\right) /(1-z)^{n}\right)_{+}\right)_{+}=\left(\prod_{i=1}^{r+1}\left(1-z^{d_{i}}\right) /(1-z)^{n}\right)_{+} \cdot
$$

This gives the following equivalent formulation of Conjecture 1

Conjecture 3. If $f_{1}, \ldots, f_{r}$ are generic forms, then $k\left[x_{1}, \ldots, x_{n}\right] /\left(f_{1}, \ldots, f_{r}\right)$ has the MRP.

An algebra $A$ has the weak Lefschetz property (WLP) if the multiplication $A_{i} \stackrel{l}{\rightarrow} A_{i+1}$ has maximal rank for all $i$ if $l$ is a generic linear form, and it has the strong Lefschetz property (SLP) if $A_{i} \stackrel{l^{d}}{\rightarrow} A_{i+d}$ has maximal rank for all $i$ and $d$ if $l$ is a generic linear form. If $A$ has the SLP, then $A$ has the MRP by semicontinuity, and if $A$ has the MRP, then $A$ has the WLP (set $d=1$ ).

The following is proved by Migliore, Miró-Roig, and Nagel in [26].

Theorem 6. If Conjecture 1 is true for all ideals generated by generic forms in $n$ variables, then all ideals generated by generic forms in $n+1$ variables have the $W L P$.

It is proved in [35] that monomial complete intersections has the SLP, thus the MRP. Hence $k[x, y, z] /\left(x^{d_{1}}, y^{d_{2}}, z^{d_{3}}, f\right)$, where $f$ is generic of degree $d_{4}$, has the Hilbert series from Conjecture 1, so the conjecture is proved for $r=n+1$. 
One might hope that powers of generic linear forms are sufficiently generic to prove Conjecture 1. This is not true. There is a conjecture due to Iarrobino [21] on when this is true (see [9]).

Conjecture 4. Let $f_{1}, \ldots, f_{r}$ be generic forms of degree $d$ and let $l_{1}, \ldots, l_{r}$ be generic linear forms. For all $(d, n, r)$ except $r=n+2, r=n+3,(n, r)=$ $(3,7),(3,8),(4,9),(5,14)$, the algebras $k\left[x_{1}, \ldots, x_{n}\right] /\left(l_{1}^{d}, \ldots, l_{r}^{d}\right)$ and $k\left[x_{1}, \ldots, x_{n}\right] /$ $\left(f_{1}, \ldots, f_{r}\right)$ have the same Hilbert series.

Some, but not all, counterexamples can be explained by the relation between ideals generated by powers of linear forms and ideals of fat points via apolarity using the famous list of counterexamples by Alexander and Hirschowitz to the expected Hilbert series of 2-fat points [1. Using [1, Alessandro Oneto (private communication) has proved that the only cases when $d$-th powers of linear forms have unexpected linear syzygies are when $(d, n, r)=(2,5,7),(3,3,5),(3,5,7)$, $(3,4,9),(3,5,14)$.

Question 2.13. Do d-th powers of generic linear forms have the same Hilbert series as the same number of generic forms of degree $d$ if $d \gg 0$ ?

Question 2.14. In connection to Conjecture 4, can one at least prove that for fixed $n$ there are only finitely many $d$ for which the series are different? If $d$ is fixed, are there finitely many $n$ for which the series are different?

Question 2.15. Let $f_{i}$ be generic forms of degree $d_{i}$ and let $l_{i}$ be generic linear forms. Do $k\left[x_{1}, \ldots, x_{n}\right] /\left(l_{1}^{d_{1}}, \ldots, l_{s}^{d_{s}}, f_{s+1}, \ldots, f_{r+s}\right)$ and $k\left[x_{1}, \ldots, x_{n}\right] /\left(f_{1}, \ldots, f_{r+s}\right)$ have the same Hilbert series if $s \leq n$ ? This is conjectured in [27]. Notice the relationship with Question 2.1.

Question 2.16. Consider the $2^{n-1}$ linear forms $l_{1}, \ldots, l_{2^{n-1}}$ in $k\left[x_{1}, \ldots, x_{n}\right]$ which are the sum of an odd number of variables. Does $k\left[x_{1}, \ldots, x_{n}\right] /\left(l_{1}^{d}, \ldots, l_{2^{n-1}}^{d}\right)$ have the same Hilbert series as $k\left[x_{1}, \ldots, x_{n}\right] / I, I$ generated by $2^{n-1}$ generic forms of degree $d$ if $n \leq 6$ ? It is true for $n \leq 4$, false for $n=7$.

2.3. Gröbner bases. A monomial ideal $I$ is called almost degrevlex if $m \in I$ implies that $m^{\prime} \in I$ for all monomials $m^{\prime}$ larger than $m$ in degrevlex with $\operatorname{deg} m^{\prime}=$ $\operatorname{deg} m$. The following conjecture is given by Moreno-Socías in [27].

Conjecture 5. The initial monomial in degrevlex order of an ideal generated by generic forms is almost degrevlex.

It is proved in [30] that Conjecture 5 implies Conjecture 1 It is also shown in [30] that the following conjecture is equivalent to Conjecture 1

Conjecture 6. If $I=\left(f_{1}, \ldots, f_{n}\right)$ is generated by generic elements in $k\left[x_{1}, \ldots, x_{n}\right]$, then multiplication with $x_{n-i}$ on $k\left[x_{1}, \ldots, x_{n}\right] /\left(\operatorname{gin}(I), x_{n}, x_{n-1}, \ldots, x_{n-i+1}\right)$ has maximal rank for $i=0, \ldots, n-1$.

2.4. Fewnomials. Monomial and binomial ideals are far from being general enough, but they are easy to work with. 
Question 2.17. For which $r$ are there monomials $m_{1}, \ldots, m_{r}$ of degree $d$ making the algebra $k\left[x_{1}, \ldots, x_{n}\right] /\left(m_{1}, \ldots, m_{r}\right)$ satisfy Conjecture 1 ?

Question 2.18. Given $d_{1}, \ldots, d_{r}$, which is the minimal Hilbert series of $k\left[x_{1}, \ldots, x_{n}\right] /\left(m_{1}, \ldots, m_{r}\right)$, each $m_{i}$ a monomial of degree $d_{i}$ ?

Question 2.19. What is the answer if we replace monomials by binomials in Questions 2.17 and 2.18? A mix between binomials and monomials? Does it, for each $r$, exist a binary ideal such that the Hochster-Laksov result holds?

\section{The TENSOR ALGEBRA}

We will denote the tensor algebra over an $n$-dimensional $k$-vector space with $k\left\langle x_{1}, \ldots, x_{n}\right\rangle$ and a two-sided ideal generated by $f_{1}, \ldots, f_{r}$ with $\left(f_{1}, \ldots, f_{r}\right)$. It is shown by Govorov [16] that if the $f_{i}$ 's are monomials, then the Hilbert series $k\left\langle x_{1}, \ldots, x_{n}\right\rangle /\left(f_{1}, \ldots, f_{r}\right)$ is a rational function, and he conjectured [17] that the same is true if the $f_{i}$ 's are homogeneous. This was shown to be false by Shearer in [34]. It is shown in [14] that even if the number of generators and the number of relations and their degrees are fixed, there is in general an infinite number of Hilbert series, and that there is no open set, even in the usual Euclidean sense with $k=\mathbb{R}$, in the space of coefficients for which the Hilbert series is constant. The smallest counterexample we know of is for three relations of degree 2 in $k\left\langle x_{1}, x_{2}, x_{3}, x_{4}\right\rangle$. It is shown in [14] that $\mathbb{R}\left\langle x_{1}, x_{2}, x_{3}, x_{4}\right\rangle /\left(x_{1} x_{2}-x_{1} x_{3}, x_{2} x_{3}-x_{3} x_{2}-x_{2}^{2} / q, x_{2} x_{4}\right)$, where $q \in \mathbb{N}_{+}$, has series $\left(1-4 z+3 z^{2}-z^{q+3}\right)^{-1}$ and that $\mathbb{R}\left\langle x_{1}, x_{2}, x_{3}, x_{4}\right\rangle /\left(x_{1} x_{2}-\right.$ $\left.x_{1} x_{3}, x_{2} x_{3}-x_{3} x_{2}, x_{2} x_{4}\right)$ has series $\left(1-4 z+3 z^{2}\right)^{-1}$. It follows that the set of algebras with three relations of degree two with series $\left(1-4 z+3 z^{2}\right)^{-1}$ is dense, but not open in $\mathbb{R}^{48}$, and that there are infinitely many series with three relations of degree two in four variables.

The remaining part of this section is taken from two papers by Anick, [2] and [3]. We restrict to the case when $\operatorname{deg}\left(x_{i}\right)=1$ for all $i$. In Anick's papers also $\operatorname{deg}\left(x_{i}\right)>1$ is treated. For two power series $A(z)=\sum_{i=0}^{\infty} a_{i} z^{i}$ and $B(z)=\sum_{i=0}^{\infty} b_{i} z^{i}$ Anick defines $A(z)<_{\operatorname{lex}} B(z)$ if for some $n$ we have $a_{i}=b_{i}$ if $i<n$ and $a_{n}<b_{n}$. It is shown in 3 that for fixed $n, d_{1}, \ldots, d_{r}$ the set $S$ of Hilbert series of algebras $k\left\langle x_{1}, \ldots, x_{n}\right\rangle /\left(f_{1}, \ldots, f_{r}\right), \operatorname{deg}\left(f_{i}\right)=d_{i}$ is well ordered. Then $\inf (S)$ is called the generic series, and an algebra with generic series is called generic. It is not clear that a generic algebra exists, and, if it does, it may very well depend on the field $k$. If the field is very large (e.g. contains the algebraic closure of an infinite transcendental extension of its prime field) it is shown that generic algebras do exist.

If $R=k\left\langle x_{1}, \ldots, x_{n}\right\rangle / I$ is a graded quotient and $f \in R_{d}$, then coefficientwise $R /(f)(z) \geq R(z)\left(1+z^{d} R(z)\right)^{-1}$. If there is equality $f$ is called strongly free (or inert) [2]. This gives that if $f_{1}, \ldots, f_{r}$ are elements in $k\left\langle x_{1}, \ldots, x_{n}\right\rangle, f_{i} \in$ $k\left\langle x_{1}, \ldots, x_{n}\right\rangle_{d_{i}}$, then the inequality

$$
k\left\langle x_{1}, \ldots, x_{n}\right\rangle /\left(f_{1}, \ldots, f_{r}\right)(z) \geq\left(\left(1-n z+z^{d_{1}}+\cdots+z^{d_{r}}\right)^{-1}\right)_{+}
$$

holds coefficientwise, and if the Hilbert series equals $\left(1-n z+z^{d_{1}}+\cdots+z^{d_{r}}\right)^{-1}$, $\left\{f_{1}, \ldots, f_{r}\right\}$ is called a strongly free set. (This is shown to be equivalent to $k\left\langle x_{1}, \ldots, x_{n}\right\rangle /\left(f_{1}, \ldots, f_{r}\right)$ being of global dimension $\leq 2$.) If one is able to find 
an algebra $k\left\langle x_{1}, \ldots, x_{n}\right\rangle /\left(f_{1}, \ldots, f_{r}\right), \operatorname{deg}\left(f_{i}\right)=d_{i}$ with Hilbert series $((1-n z+$ $\left.\left.z^{d_{1}}+\cdots+z^{d_{r}}\right)^{-1}\right)_{+}$, this algebra is generic. The concept of strongly free is the counterpart to regular sequences in the commutative case. It is tempting to conjecture that for any set of forms $g_{1}, \ldots, g_{r}$ with $g_{i} \in k\left\langle x_{1}, \ldots, x_{n}\right\rangle_{d_{i}}$, we have $k\left\langle x_{1}, \ldots, x_{n}\right\rangle /\left(g_{1}, \ldots, g_{r}\right)(z) \geq\left(\left(1-n z+z^{d_{1}}+\cdots+z^{d_{r}}\right)^{-1}\right)_{+}$, and that there is some set $\left\{g_{1}, \ldots, g_{r}\right\}$ which gives equality. The inequality is proved in [3]. There it is also shown that if $\operatorname{deg}\left(g_{1}\right)=2, \operatorname{deg}\left(g_{2}\right)=d \geq 7$, there is no algebra $k\left\langle x_{1}, x_{2}\right\rangle /\left(g_{1}, g_{2}\right)$ with series $\left(\left(1-2 z+z^{2}+z^{d}\right)^{-1}\right)_{+}$, so the second part of the statement is not true.

However, the only known examples for when the series for $k\left\langle x_{1}, \ldots, x_{n}\right\rangle /\left(f_{1}, \ldots\right.$, $\left.f_{r}\right)$ does not equal that for $\left.\left(1-n z+z^{d_{1}}+\cdots+z^{d_{r}}\right)^{-1}\right)+$ is when $k\left\langle x_{1}, \ldots, x_{n}\right\rangle /\left(f_{1}\right.$, $\left.\ldots, f_{r}\right)$ is artinian. When $k\left\langle x_{1}, \ldots, x_{n}\right\rangle /\left(f_{1}, \ldots, f_{r}\right)$ is not artinian, Anick indicates that one should expect the series to be equal to $\left(1-n z+z^{d_{1}}+\cdots+z^{d_{r}}\right)^{-1}$.

In case $\operatorname{deg}\left(f_{i}\right)=2$ for all $i$, one can say a bit more. If $r \leq n^{2} / 4$ there are strongly free sets, so in this case the generic series is $\left(1-n z+r z^{2}\right)^{-1}$ [3. Lemma 5.10]. If $r \geq n^{2} / 2$ there exists an algebra with series $\left(\left(1-n z+r z^{2}\right)^{-1}\right)_{+}$.

We conclude this section with some open problems. The first two concern forms of degree 2, while the last two are of more general nature. Also cf. [3].

Question 3.1. Consider $r$ generic forms of degree 2. Is the generic series equal to $\left(1 /\left(1-n z+r z^{2}\right)\right)+$ also if $n^{2} / 4<r<n^{2} / 2$ ?

Question 3.2. Consider again $r$ generic forms of degree 2. For which $r$ is the generic algebra artinian?

Question 3.3. Let $f_{1}, \ldots, f_{r}$ be generic forms of degrees $d_{1}, \ldots, d_{r}$ and suppose that the algebra $k\left\langle x_{1}, \ldots, x_{n}\right\rangle /\left(f_{1}, \ldots, f_{r}\right)$ is artinian. Can one give a better lower bound for the series than $\left(\left(1-n z+z^{d_{1}}+\cdots+z^{d_{r}}\right)^{-1}\right)_{+}$?

Question 3.4. Let $f_{1}, \ldots, f_{r}$ be generic forms of degrees $d_{1}, \ldots, d_{r}$. Is the series for the algebra $k\left\langle x_{1}, \ldots, x_{n}\right\rangle /\left(f_{1}, \ldots, f_{r}\right)$ equal in the first nontrivial degree $\min \left\{d_{i}\right\}+1$ to the series $\left(\left(1-n z+z^{d_{1}}+\cdots+z^{d_{r}}\right)^{-1}\right)_{+}$? A positive answer would be the tensor algebra analogue to the Hochster-Laksov result.

\section{LiE ALGEBRAS}

We consider $\mathbb{N}$-graded Lie algebras. The Jacobi identity is $[[a, b], c]=[a,[b, c]]+$ $[[a, c], b]$. To get quotients of associative algebras we study the universal enveloping algebra of Lie algebras. Thus we study rings of type $T(V) / I$, where $I$ is generated by Lie elements, i.e., images of elements in the Lie algebra. We know that $\left(\left(1-n z+\sum_{i=1}^{r} z^{d_{i}}\right)^{-1}\right)_{+}$, where $n$ is the number of generators and we have $r$ relations of degree $d_{1}, \ldots, d_{r}$, is a lower bound for the Hilbert series. Anick [3] gives an example, $k\langle x, y\rangle /\left(f_{1}, f_{2}\right)$, where $\operatorname{deg}\left(f_{1}\right)=3$, $\operatorname{deg}\left(f_{2}\right)=8$. $\left(f_{1}\right.$ can after a change of basis be chosen as $[x,[x, y]]=\left[x^{2}, y\right]$.) He shows that the series is not the expected $\left(1-2 z+z^{3}+z^{8}\right)^{-1}$. He also shows [2] that if all relations are of degree two, so they are linear combinations of commutators of variables, then there are strongly free sets of Lie elements of size $r$ if $r \leq n^{2} / 4$, so the Hilbert series is $\left(1-n z+r z^{2}\right)^{-1}[3$, Lemma 5.10]. 
Example If $r \leq n-1$, we can get the expected Hilbert series by choosing commutators. If $I=\left(\left[x_{1}, x_{2}\right],\left[x_{1}, x_{3}\right], \ldots,\left[x_{1}, x_{n}\right]\right)$, then $k\left\langle x_{1}, \ldots, x_{n}\right\rangle / I$ has the expected series $\left(1-n z+(n-1) z^{2}\right)^{-1}$ as a simple calculation shows.

Question 4.1. Is the generic series for $r$ generic Lie elements of degree 2 in $n$ variables equal to $\left(\left(1-n z+r z^{2}\right)^{-1}\right)_{+}$?

Question 4.2. How many generic Lie elements of degree $d$ are required for $L$ to be nilpotent?

\section{EXTERIOR ALGEBRAS}

Let $V$ be a $k$-vector space of dimension $n$ and denote by $\Lambda(V)$ the exterior algebra on $n$ generators, that is, $\Lambda(V)=k\left\langle x_{1}, \ldots, x_{n}\right\rangle /\left(x_{i} x_{j}+x_{j} x_{i}, 1 \leq j \leq n, x_{i}^{2}, i=\right.$ $1, \ldots, n)$. In the same way as for commutative algebras, one can show that there is a coefficientwise minimal series which is achieved on a non-empty Zariski open set of the coefficients, and that we have the corresponding inequality for the Hilbert series. Thus, if $I$ is an ideal generated by generic forms $f_{i}, \operatorname{deg}\left(f_{i}\right)=d_{i}, i=1, \ldots, r$, then $\Lambda(V) / I(t)$ is coefficientwise smaller or equal to $\left(\prod_{i=1}^{r}\left(1-z^{d_{i}}\right)(1+z)^{n}\right)_{+}$. A first guess could be that if $I$ is an ideal generated by generic forms, then $\Lambda V / I(z)=$ $k\left[x_{1}, \ldots, x_{n}\right] /\left(\left(x_{1}^{2}, \ldots, x_{n}^{2}\right)+J\right)(z)$, where $J$ has "the same" generators as $I$. It is true for a principal ideal generated by an element of even degree [28, so if $f$ is a generic element of even degree $d$, then the Hilbert series is $\left(\left(1-z^{d}\right)(1+z)^{n}\right)_{+}$. But since $f \subseteq \operatorname{Ann} f$ when $f$ is odd, the guess is not true even for principal ideals.

In [22, the series for $\Lambda(V) /(f)$ is determined for $d$ odd and equal to $n-2$ (the expected series $+t^{n-1}$ ) and $n-3$ (the expected series) and for $(9,3)$ (the expected series $\left.+3 t^{6}\right)$. A lower bound for the series for any $d$ is also given, providing a counterexample to Conjecture 6.1 in 28 .

When the ideal is generated by two generic quadratic forms, it was noticed in 15. that for $n=5$, the Hilbert series differs from the expected series by one in degree three. The following conjecture, providing a combinatorial description of the series, is given in [10].

Conjecture 7. Let $f$ and $g$ be generic quadratic forms. Then the Hilbert series of $\Lambda(V) /(f, g)$ is equal to $1+a(n, 1) t+a(n, 2) t^{2}+\cdots+a(n, s) t^{s}+\cdots$, where $a(n, s)$ is the number of lattice paths inside the rectangle $(n+2-2 s) \times(n+2)$ from the bottom left corner to the top right corner with moves of two types: $(x, y) \rightarrow(x+1, y+1)$ or $(x-1, y+1)$.

It is proved in [10], using the theory of matrix pencils, that the series in Conjecture 7 is an upper bound for $\Lambda(V) /(f, g)$.

Question 5.1. Let $l_{1}$ and $l_{2}$ be generic linear forms in $k\left[x_{1}, \ldots, x_{n}\right]$. Does the Hilbert series of $k\left[x_{1}, \ldots, x_{n}\right] /\left(x_{1}^{2}, \ldots, x_{n}^{2}, l_{1}^{2}, l_{2}^{2}\right)$ equal that for $\Lambda(V) /(f, g), f$ and $g$ generic quadratic forms? This is conjectured in [10]. The corresponding question for three generic linear forms is not true.

Question 5.2. The Hochster-Laksov result does not hold in the exterior algebra when $n=5, d_{1}=d_{2}=2$, as shown in [15]. Is this the only counterexample? 
Question 5.3. Consider a supercommutative algebra, generated by even and odd elements. In this setting the usual commutative algebra is a superalgebra with even generators, while the exterior algebra is a superalgebra with odd generators. Given a degree sequence $\left(d_{1}, \ldots, d_{r}\right)$, what is the minimal Hilbert series that such an algebra can have? If at most half of the elements are odd?

\section{Bigraded ALGEBRAS}

The coordinate ring of $\mathbb{P}^{m} \times \mathbb{P}^{n}$ is $R=k\left[x_{0}, \ldots, x_{m}, y_{0}, \ldots, y_{n}\right]$, where $\operatorname{deg} x_{i}=$ $(1,0)$ and $\operatorname{deg} y_{i}=(0,1)$. We propose the problem to determine the Hilbert series of generic (bigraded) ideals in $R$. Suppose that the ideal is generated by $f_{1}, \ldots, f_{r}, \operatorname{deg} f_{i}=\left(d_{i}, e_{i}\right)$. A natural guess is that the bigraded Hilbert series of $R /\left(f_{1}, \ldots, f_{r}\right)$ is

$$
\left(\prod_{i=1}^{r}\left(1-x^{d_{i}} y^{e_{i}}\right) /\left((1-x)^{2}(1-y)^{2}\right)\right)_{+},
$$

where $\left(\sum_{i, j \geq 0} a_{i, j} x^{i} y^{j}\right)_{+}=\sum b_{i, j} x^{i} y^{j}$, where $b_{i, j}=a_{i, j}$ if $a_{k, l}>0$ for all $(k, l) \leq$ $(i, j)$ and $b_{i, j}=0$ otherwise. The guess is not true in general; the first counterexample that we have found is for three relations of degree $(2,1)$ in the coordinate ring of $\mathbb{P}^{1} \times \mathbb{P}^{2}$. But we conjecture that it is true when $m=n=1$.

Conjecture 8. If $f_{1}, \ldots, f_{r}$ are generic bigraded forms, $\operatorname{deg} f_{i}=\left(d_{i}, e_{i}\right)$, in $R=$ $k\left[x_{0}, x_{1}, y_{0}, y_{1}\right]$, then the Hilbert series of $R /\left(f_{1}, \ldots, f_{r}\right)$ is equal to $\left(\prod_{i=1}^{r}(1-\right.$ $\left.\left.\left.x^{d_{i}} y^{e_{i}}\right) /\left((1-x)^{2}(1-y)^{2}\right)\right)\right)_{+}$.

We have checked the conjecture on computer in case all relations are of the same degree $(d, e) \leq(3,3)$ and in case all degrees are of the form $\left(d_{i}, e_{i}\right)$ with $d_{i}+e_{i}=3$, and the corresponding claim in a few examples in the coordinate ring of $\mathbb{P}^{1} \times \mathbb{P}^{1} \times \mathbb{P}^{1}$.

We conclude with some open questions.

Question 6.1. If $f_{1}, \ldots, f_{r}$ are generic multigraded forms in the coordinate ring $R$ of $\mathbb{P}^{1} \times \cdots \times \mathbb{P}^{1}=\left(\mathbb{P}^{1}\right)^{n}, \operatorname{deg} f_{i}=\left(d_{1 i}, \ldots, d_{n i}\right)$, is the Hilbert series of $R /\left(f_{1}, \ldots, f_{r}\right)$

$$
\left(\prod_{i=1}^{r}\left(1-x_{1}^{d_{1 i}} \cdots x_{n}^{d_{n i}}\right) / \prod_{i=1}^{n}\left(1-x_{i}\right)^{2}\right)_{+} ?
$$

Question 6.2. What is the Hilbert series of an ideal generated by generic bigraded forms in the coordinate ring of $\mathbb{P}^{m} \times \mathbb{P}^{n}$ ?

\section{Positive Characteristic}

Conjecture 1 is true for any field when $n \leq 2$, [12. When $n=3$, it is true when $k$ is infinite [4]. The Hochster-Laksov result [19], that Conjecture 1 is correct up to the first non-trivial degree, is not dependent upon the characteristics, but on the field being algebraically closed.

We believe that Conjecture 1 is true for any field. However, some of the questions that we have posed in the previous sections have a negative answer. 
Let us for instance consider Question 2.1. Let $r=n+1$ and consider uniform degrees of the generators. It is well known that the Hilbert function of a CI with forms of degree $d$ is symmetric around $n(d-1) / 2$. Now consider characteristic $p \geq d$. Then $f_{r+1}^{p-1} \cdot f_{r+1}=f_{r+1}^{p}=0$ in $k\left[x_{1}, \ldots, x_{n}\right] /\left(x_{1}^{d}, \ldots, x_{n}^{d}\right)$, so if $\left(d^{p-1}+d^{p}\right) / 2 \leq$ $n(d-1) / 2$, the series is not the one given in Conjecture 1 For $p=d=2$, this happens when $n \geq 6$.

Question 7.1. Does the Hochster-Laksov result hold when $k$ is finite?

Question 7.2. Is Conjecture 1 true when $n=3$ and $k$ is finite?

Question 7.3. Stanley used an explicit choice of forms when proving Conjecture 1 for $r=n+1$. As we have seen above, this choice does not work in positive characteristic. Can one give a construction valid also for positive characteristic?

\section{ACKNOWLEDGEMENTS}

We thank the participants of the Stockholm problem solving seminar for many fruitful discussions related to the questions and conjectures presented in this paper.

\section{REFERENCES}

[1] Alexander, J. and Hirschowitz, A. Polynomial interpolation in several variables, J. Algebraic Geom. 4 (1995), no. 2, 201-222. MR 1311347

[2] Anick, D. Non-commutative graded algebras and their Hilbert series, J. Algebra 78 (1982), no. 1, 120-140. MR 0677714

[3] Anick, D. Generic algebras and CW complexes. In: Algebraic topology and algebraic $K-$ theory (Princeton, N.J., 1983), 247-321, Ann. of Math. Stud. 113, Princeton Univ. Press, Princeton, NJ, 1987. MR 0921481

[4] Anick, D. Thin algebras of embedding dimension three, J. Algebra 100 (1986), no. 1, 235-259. MR 0839581

[5] Aubry, M. Série de Hilbert d'une algèbre de polynômes quotient, J. Algebra 176 (1995), no. 2, 392-416. MR 1351616

[6] Backelin, J. and Oneto, A. On a class of power ideals, J. Pure Appl. Algebra 219 (2015), no. 8, 3158-3180. MR 3320212

[7] Boij, M., Fröberg, R., and Lundqvist, S., Powers of generic ideals and the weak Lefschetz property for powers of some monomial complete intersections, J. Algebra 495 (2018), 1-14. MR 3726101

[8] Catalisano, M. V., Chiantini, L., Geramita, A., and Oneto, A. Waring-like decompositions of polynomials, 1, Linear Algebra Appl. 533 (2017), 311-235. MR 3695910.

[9] Chandler, K. The geometric interpretation of Fröberg-Iarrobino conjectures on infinitesimal neighbourhoods of points in projective space, J. Algebra 286 (2005), no. 2, 421-455. MR 2128025.

[10] Crispin Quiñonez, V., Lundqvist, S. and Nenashev, G., On ideals generated by two generic quadratic forms in the exterior algebra. Preprint, arXiv:1803.08918 [math.AC]

[11] Deery, T. Rev-lex segment ideals and minimal Betti numbers. In: The Curves Seminar at Queen's, Vol. X (Kingston, ON, 1995), 193-219. Queen's Papers in Pure and Appl. Math., 102, Queen's Univ., Kingston, ON, 1996. MR 1381739. 
[12] Fröberg, R. An inequality for Hilbert series, Math. Scand. 56 (1985), no. 2, 117-144. MR 0813632

[13] Fröberg, R. and Hollman, J. Hilbert series for ideals generated by generic forms, J. Symbolic Comput. 17 (1994), no. 2, 149-157. MR 1283740

[14] Fröberg, R. and Löfwall, C. On Hilbert series for commutative and noncommutative graded algebras, J. Pure Appl. Algebra 76 (1991), no. 1, 33-38. MR 1140638

[15] Fröberg, R. and Löfwal, C. Koszul homology and Lie algebras with application to generic forms and points, Homology Homotopy Appl. 4 (2002), no. 2, 227-258. MR 1918511

[16] Govorov, V. E. Graded algebras, Math. Notes 12 (1972), 552-556. MR 0318199

[17] Govorov, V. E. The dimension of graded algebras, Math. Notes 14 (1973), 678-682. MR 0332876

[18] Grayson, D. R. and Stillman, M. E. Macaulay 2, a software system for research in algebraic geometry. https://faculty.math.illinois.edu/Macaulay2/

[19] Hochster, M. and Laksov, D. The linear syzygies of generic forms, Comm. Algebra 15 (1987), no. 1-2, 227-239. MR 0876979

[20] Iarrobino, A. Compressed algebras: Artin algebras having given socle degrees and maximal length, Trans. Amer. Math. Soc. 285 (1984), no. 1, 337-378. MR 0748843

[21] Iarrobino, A. Inverse system of a symbolic power, III. Thin algebras and fat points, Compositio Math. 108 (1997), no. 3, 319-356. MR 1473851

[22] Lundqvist, S., Nicklasson, L., On generic principal ideals in the exterior algebra. Preprint, arXiv:1803.03563 [math.AC]

[23] Macaulay, F. Some properties of enumeration in the theory of modular systems, Proc. London Math. Soc. (2) 26 (1927), 531-555. MR 1576950

[24] Migliore, J. and Miró-Roig, R. M. Ideals of general forms and the ubiquity of the weak Lefschetz property, J. Pure Appl. Algebra 182 (2003), no. 1, 79-107. MR 1978001

[25] Migliore, J. and Miró-Roig, R. M. On the minimal free resolution of $n+1$ general forms, Trans. Amer. Math. Soc. 355 (2003), no. 1, 1-36. MR 1928075

[26] Migliore, J., Miró-Roig, R. M. and Nagel, U. On the weak Lefschetz property for powers of linear forms, Algebra Number Theory 6 (2012), no. 3, 487-526. MR 2966707

[27] Moreno-Socías, G. Degrevlex Gröbner bases of generic complete intersections, J. Pure Appl. Algebra 180 (2003), no. 3, 263-283. MR 1966660

[28] Moreno-Socías, G. and Snellman, J. Some conjectures about the Hilbert series of generic ideals in the exterior algebra, Homology Homotopy Applications 4 (2002), no. 2, 409-426. MR 1918519

[29] Nenashev, G. A note on Fröberg's conjecture for forms of equal degrees, C. R. Math. Acad. Sci. Paris 355 (2017), no. 3, 272-276. MR 3621254

[30] Pardue, K. Generic sequences of polynomials, J. Algebra 324 (2010), no. 4, 579-590. MR 2651558

[31] Pardue, K. and Richert, B. Syzygies of semi-regular sequences, Illinois J. Math. 53 (2009), no. 1, 349-364. MR 2584951.

[32] Pardue, K. and Richert B, Errata for Syzygies of semi-regular sequences, Illinois J. Math. 56 (2012), no. 4, 1001-1003. MR 3231471.

[33] Nicklasson, L. On the Hilbert series of ideals generated by generic forms, Comm. Algebra 45 (2017), no. 8, 3390-3395. MR 3609345.

[34] Shearer, J. B. A graded algebra with non-rational Hilbert series, J. Algebra 62 (1980), no. 1, 228-231. MR 0561125 
[35] Stanley, R. Hilbert functions of graded algebras, Advances in Math. 28 (1978), no. 1, 57-83. MR 0485835

[36] Watanabe, J. The Dilworth number of Artinian rings and finite posets with rank function. In: Commutative algebra and combinatorics (Kyoto, 1985), 303-312, Adv. Stud. Pure Math., 11, North-Holland, Amsterdam, 1987. MR 0951211

\section{R. Fröberg}

Department of Mathematics, Stockholm University, S-10691, Stockholm, Sweden

ralff@math.su.se

S. Lundqvist $\bowtie$

Department of Mathematics, Stockholm University, S-10691, Stockholm, Sweden

samuel@math.su.se

Received: December 5, 2017

Accepted: April 27, 2018 